\title{
Endourologic Management of an latrogenic Ureteral Avulsion Using a Thermoexpandable Nickel-Titanium Alloy Stent (Memokath 051)
}

\author{
Maral J. Rouhani, MRCS, Hamid Abboudi, MRCS, Norma Gibbons, FRCS MD, \\ and Tamer El-Husseiny, FRCS MD
}

\begin{abstract}
Background: The use of ureteroscopy in the management of urolithiasis is well established. Ureteral avulsion is a rare but challenging complication of the procedure. Postureteral injury strictures are a common result of such injuries and are typically managed with reconstructive surgery or endoscopically with polymer stent. This case represents the first effective management of ureteral avulsion and subsequent ureteral stricture using a Memokath ureteral stent. Case Presentation: A 54-year-old gentleman presented to the Department of Urology with right loin pain as a result of right renal calculi, previously treated with extracorporeal shockwave lithotripsy. The patient was investigated with ultrasonography and noncontrast CT of his urinary tract, revealing mild right-sided hydroureteronephrosis and two right proximal ureteral stones, measuring 9 and $4 \mathrm{~mm}$, respectively. He underwent a right semirigid ureteroscopy and laser stone fragmentation with complete stone clearance, but on withdrawal of the ureteroscope, a right ureteral injury occurred with ureteral mucosal avulsion extending from the L3/L4 vertebrae to the right vesicoureteral junction. Upon consideration of several options for management of this ureteral avulsion, the patient opted for endourologic stenting. After 10 months, the patient developed a ureteral stricture as a result of the avulsion. He was troubled with stent-related symptoms and wanted to avoid reconstructive surgery and, therefore, opted for a Memokath ureteral stent. The patient recovered well with excellent renal function and drainage on subsequent mercaptoacetyl-triglycyl renogram.

Conclusion: Ureteral avulsion is a rare but important complication of ureteroscopy with numerous options available for management. Discussions should be had with the patient to weigh the various options, and metallic stents should be considered in the long-term management of such injuries and their sequel.
\end{abstract}

Keywords: ureteral injury, ureteroscopy, stent

\section{Introduction}

$\mathbf{U}$ ROLITHIASIS IS A common urologic presentation, and intervention using ureteroscopy has become one of the main modalities of management. Ureteral avulsion is a recognized but rare complication of ureteroscopy. We present a case of management of ureteral avulsion using a Memokath ureteral stent.

\section{Case Report}

A 54-year-old gentleman presented to our specialized stone clinic in October 2015 with right loin pain, on a back- ground of previous extracorporeal shockwave lithotripsy for right renal stones. His medical history also included hypertension and hypercholesterolemia. Ultrasonography of his urinary tract revealed mild right-sided hydronephrosis and a subsequent noncontrast CT confirmed the presence of two right proximal ureteral stones, measuring 9 and $4 \mathrm{~mm}$, respectively, with associated mild hydroureteronephrosis.

He underwent a right semirigid ureteroscopy and laser stone fragmentation with complete stone clearance. On withdrawal of the ureteroscope at the end of the procedure, a right ureteral injury occurred with ureteral mucosal avulsion extending from the level L3/L4 vertebrae to the right

Department of Urology, Charing Cross Hospital, London, United Kingdom.

(C) Maral Rouhani et al. 2017; Published by Mary Ann Liebert, Inc. This is an Open Access article distributed under the terms of the Creative Commons Attribution License, which permits unrestricted use, distribution, and reproduction in any medium, provided the original work is properly cited. Mary Ann Liebert, Inc. offers reprint services for those who want to order professionally produced copies of articles published under the Creative Commons Attribution (CC BY) license. To obtain a price quote, email Reprints@liebertpub.com. Please include the article's title or DOI, quantity, and delivery destination in your email. 
FIG. 1. Retrograde study confirming disruption to the course of the ureter with inability to place a guidewire into the kidney (A). A Double-J polymeric stent was inserted to preserve the patency of the mid and distal ureter $(\mathbf{B})$.
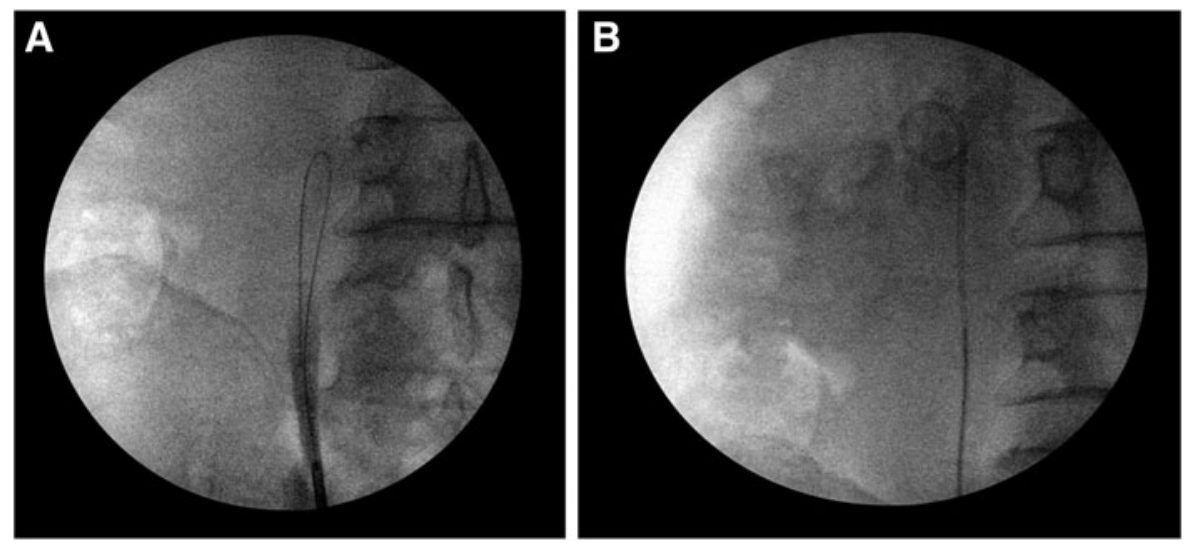

vesicoureteral junction. Ureteroscopic-guided attempts at insertion of a guidewire could not breach the entire ureteral defect; therefore, a $6 \mathrm{~F} 26 \mathrm{~cm}$ ureteral stent was inserted to ensure that the ureteral lumen is preserved because the ureter was completely avulsed with no overlying mucosa (Fig. 1). An urgent nephrostomy was inserted by the interventional radiologist after an urgent CT urogram (Fig. 2).

Several management options were urgently considered in a multidisciplinary team approach, including urologic surgeons, renal transplant surgeons, and interventional radiologists. Options included endoscopic management, ureteral reconstruction by either a Boari flap or an ileal interposition, autotransplantation, transureteroureterostomy, and finally even consideration of a nephrectomy.

The patient and his family opted for endoscopic management, so on day 1 postoperatively, a combined antegrade/ retrograde endoscopic repair (rendezvous procedure) was performed effectively, with insertion of an $8 \mathrm{~F} 26 \mathrm{~cm}$ rightsided ureteral stent to bridge the segment of the avulsed ureter (Fig. 3). He recovered well postoperatively with normalized blood tests and good urine output. He was discharged on day 5 after the initial ureteroscopy with a nephrostomy and urethral catheter both on free drainage. He attended for a weekly consultant urologist outpatient review.

A nephrostogram was performed 3 weeks later that showed satisfactory passage of contrast from the renal pelvis to the bladder with no evidence of ureteral leak. The nephrostomy was clamped and the urinary catheter was subsequently removed. A dimercaptosuccinic acid (DMSA) nuclear medicine scan was also performed at 3 weeks, which showed homogeneous distribution of tracer throughout both kidneys, at $44 \%$ for the right kidney and $56 \%$ for the left kidney.

At week 6, further retrograde and antegrade studies were performed along with a right semirigid ureteroscopy, which showed a long stricture from the level of L3 to the vesicoureteral junction. The stent was replaced effectively with a $6 \mathrm{~F} 26 \mathrm{~cm}$ ureteral stent and the right nephrostomy was removed. The patient remained well and was seen again 3 months later for another right retrograde study and ureteroscopy. The ureteral stricture was again noted from the level of L3 down to the level of vesicoureteral junction. As such, balloon endodilatation was performed followed by insertion of an $8 \mathrm{~F} 26 \mathrm{~cm}$ stent. The patient was persistently troubled by stent-related symptoms, in particular, urinary frequency, urgency, and hematuria. A further DMSA scan 6 months after the original injury showed normal split function with no significant change from the previous study.

Given the stricture recurrence, the option of reconstructive surgery for management of the right ureteral stricture was rediscussed further, but the patient opted for endourologic management, namely a semipermanent Memokath ureteral stent to bridge the length of the stricture. At 10 months after
FIG. 2. Contrast CT showing right ureteral disruption with retroperitoneal contrast extravasation (white arrow). The proximal ureteral stent is seen within the retroperitoneum (red arrow).
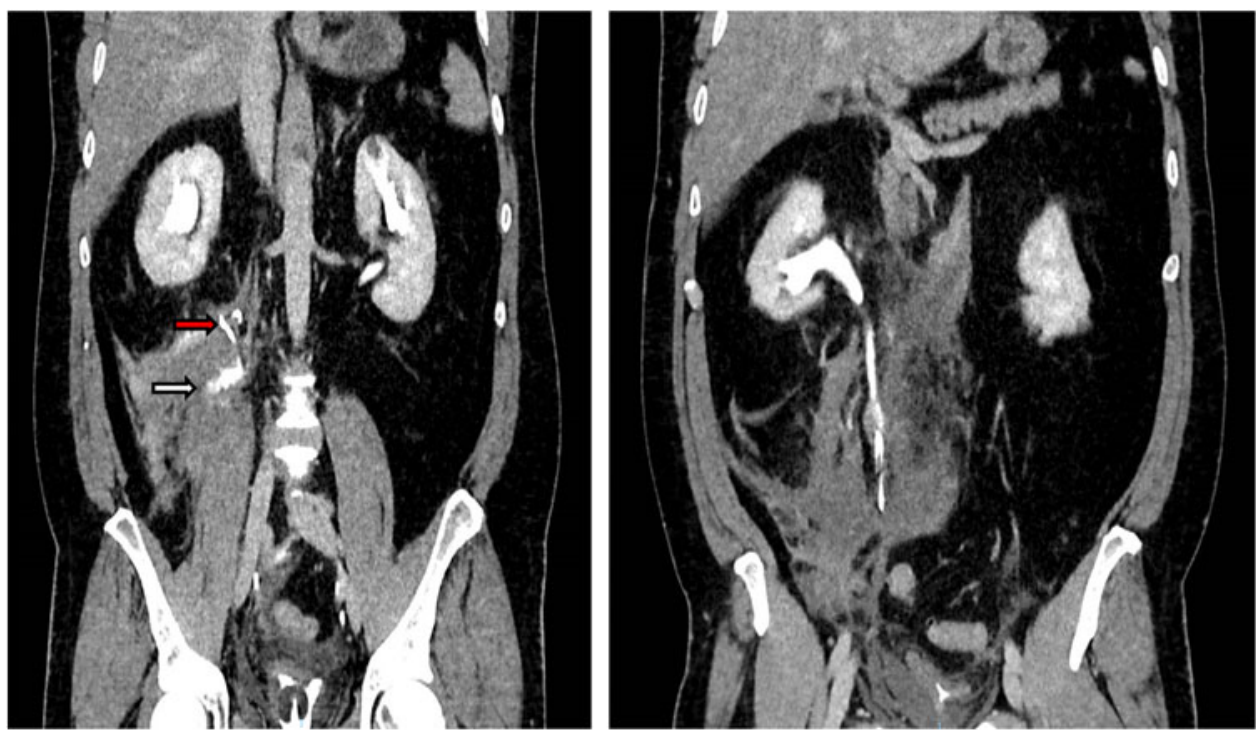

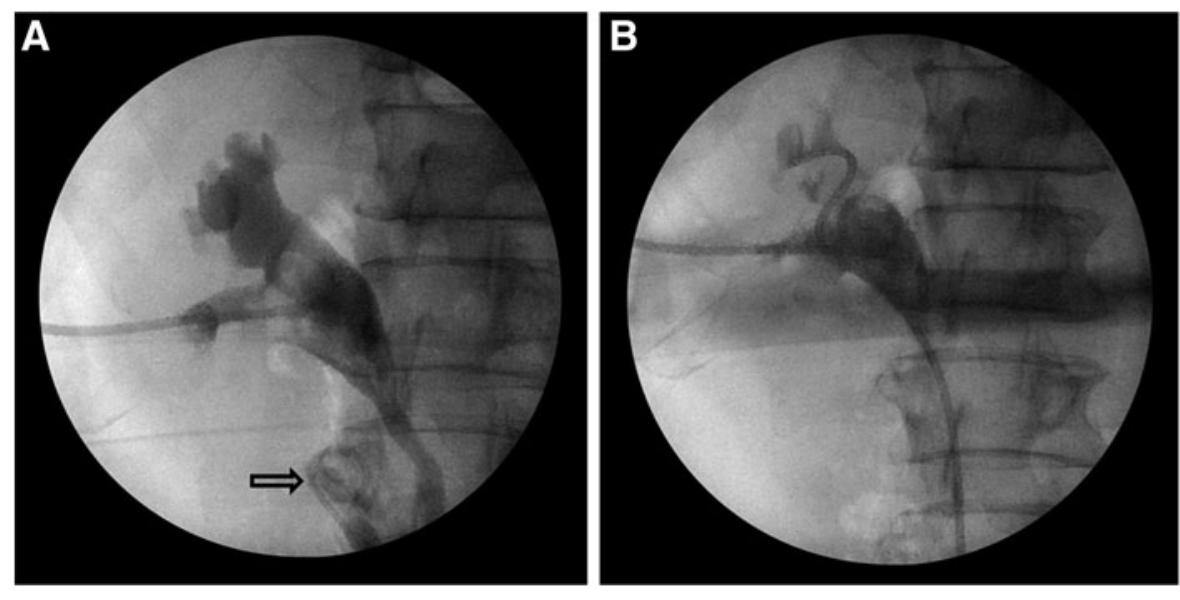

FIG. 3. Nephrostogram identifying the Double-J stent outside the course of the ureter (A) (arrow).

Followed by effective rendezvous insertion of a Double-J polymeric stent across the ureteral defect (B). the original injury, a special-order $20 \mathrm{~cm}$ dual cone Memokath was effectively inserted (Fig. 4). The patient has made good recovery and has been reporting less stent-related symptoms and a better quality of life than when using the standard Double-J stents.

A mercaptoacetyl-triglycyl (MAG3) renogram 14 months postoperatively on the December 13, 2016, showed no obstruction to drainage, with the right kidney contributing to $46 \%$ function (Fig. 4).

\section{Discussion}

This is the first reported case of the use of a Memokath stent in the management of a ureteral stricture secondary to iatrogenic ureteral avulsion. Ureteral avulsion is a rare but serious complication of ureteroscopy, the incidence of which has been reported as $0 \%-3.75 \% .^{1}$ The urologist is faced with a number of management options in such a situation, ranging from stenting to open reconstruction. Such a situation also poses a challenge in communication with the patient. It is vital that a frank discussion takes place with the patient, explaining that the entire renal unit is potentially at stake, yet there are a number of management options; the decision should then be made jointly between patient and surgeon.

The management of iatrogenic ureteral avulsion depends greatly on the site and severity of injury. For proximal injuries, end-to-end anastomosis can be considered; Boari flap and Psoas hitch are options for middle-third ureteral avulsion. For severe injuries, including total ureteral avulsion from both ends, options include ureteral implantation using an ileal interposition, renal autotransplantation, and nephrectomy. Each management option has benefits and drawbacks, and so must be tailored for suitability to the patient.

With regard to open reconstructive ureteral injury repairs, conventional teaching dictates a delayed approach to allow inflammation and extravasation to subside. However, in the endoscopic setting, an early attempt at realignment of the ureteral ends is often utilized to capitalize on the naivety of the tissue injury, exploiting the patency of the ureteral ends before the process of scaring and fibrosis of the ureter setting in.
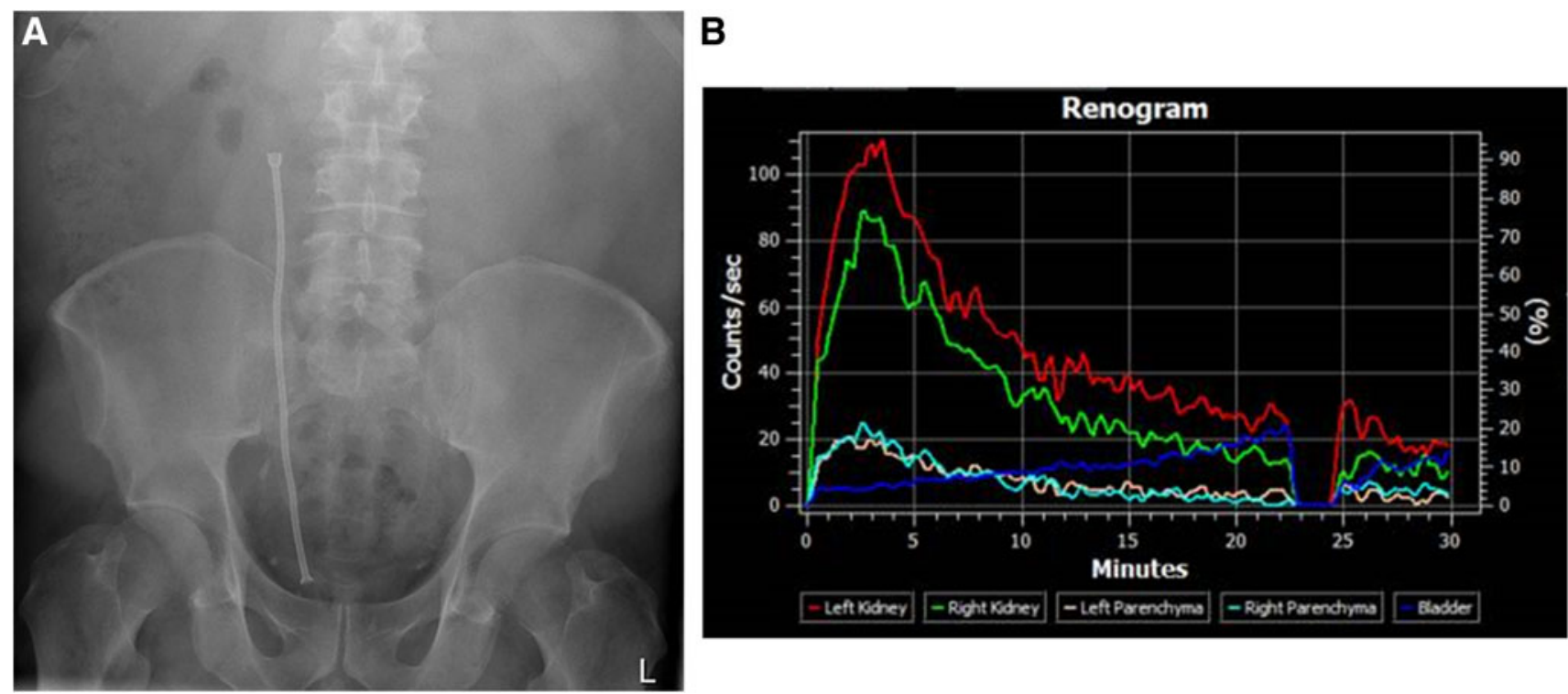

FIG. 4. Right ureteral Memokath stent bridging the strictured $4 \mathrm{~cm}$ segment of ureter that extends from the level of L4/L5 down to the level of the upper sacroiliac joint (A). A MAG 3 renogram with normal appearance and drainage of both kidneys. No evidence of obstruction seen (B). 
Double-J polymeric stents have been traditionally used to overcome strictures resulting from an avulsed ureter; however, they are associated with numerous problems: encrustation (necessitating 3-6 monthly stent changes), stone formation, pain, infection, and reflux. The use of metallic stents over long-term Double-J stents has progressed over the past 20 years, with an additional advantage of likely longer term patency because of resilience of compression; a wide variety of stents are now available. The Memokath 051 stent is a thermoexpandable titanium-nickel spiral shaped stent, ${ }^{2}$ with available lengths of 3,6,10, and $15 \mathrm{~cm}$, and patency rates from $90 \%$ to $100 \%$. It has been shown to have favorable outcomes in overcoming benign ureteral strictures, as first reported by Arya et al. ${ }^{3}$; more recently in 2014, Bourdoumis et al. ${ }^{4}$ effectively used the Memokath stent in treating obstruction secondary to retroperitoneal fibrosis.

We have shown that the Memokath 051 stent can be used effectively in treating ureteral stricture secondary to iatrogenic ureteral avulsion and it should be considered a viable option when considering the various management options.

\section{Disclosure Statement}

PNN has provided funding towards the journal's publication fee. No other disclosures.

\section{References}

1. Al-Awadi K, Kehinde EO, Al-Hunayan A, et al. Iatrogenic ureteric injuries: Incidence, aetiological factors and the effect of early management on subsequent outcome. Int Urol Nephrol 2005;37:235-241.

2. Klarskov P, Nordling J, Nielsen JB. Experience with Memokath 051 ureteral stent. Scand J Urol Nephrol 2005;39: 169-172.
3. Arya M, Mostafid H, Patel HR, et al. The self-expanding metallic ureteric stent in the long-term management of benign ureteric strictures. BJU Int 2001;88:339-342.

4. Bourdoumis A, Kachrilas S, Kapoor S, et al. The use of a thermoexpandable metal alloy stent in the minimally invasive management of retroperitoneal fibrosis: A single center experience from the United Kingdom. J Endourol 2014;28: 96-99.

Address correspondence to:

Tamer El-Husseiny, FRCS MD

Department of Urology

Charing Cross Hospital

London W6 8RF

United Kingdom

E-mail: tamer.el-husseiny@imperial.nhs.uk

Abbreviations Used

$\mathrm{CT}=$ computed tomography

DMSA $=$ dimercaptosuccinic acid

Cite this article as: Rouhani MJ, Abboudi $\mathrm{H}$, Gibbons N, El-Husseiny T (2017) Endourologic management of an iatrogenic ureteral avulsion using a thermoexpandable nickel-titanium alloy stent (Memokath 051), Journal of Endourology Case Reports 3:1, 57-60, DOI: 10.1089/ cren.2017.0019. 